\title{
Attitudes Toward Posthumous Assisted Reproduction in China: A Multi-Dimensional Survey
}

\section{Jiliang Huang}

The First Affiliated Hospital of Shantou University Medical College

Jue Li

Shenzhen Hospital of Southern Medical University

\section{Wanfen Xiao}

The First Affiliated Hospital of Shantou University Medical College

Zhiling Li ( $\sim$ stlizhiling@126.com )

The First Affiliated Hospital of Shantou University Medical College https://orcid.org/0000-0002-1467-0641

\section{Research}

Keywords: Posthumous assisted reproduction, Embryos, Attitude, Offspring, Ethics

Posted Date: November 2nd, 2021

DOI: https://doi.org/10.21203/rs.3.rs-1016388/v1

License: (a) (i) This work is licensed under a Creative Commons Attribution 4.0 International License. Read Full License 


\section{Abstract \\ Background}

Professional legislation and ethics guidelines for posthumous assisted reproduction (PAR) are lacking in China. This study aims to measure the attitudes of the general public, IVF couples, and ART practitioners toward PAR in China.

\section{Methods}

A multi-dimensional survey was designed, and electronic questionnaires were used. General demographic data, reproductive viewpoints, attitudes toward PAR, interactive predictive attitudes in couples, and the legal attribute and disposition right of posthumous embryos were evaluated.

\section{Results}

The study found that the traditional Chinese viewpoints of fertility had changed. The approval rates for PAR were $79.10 \%, 55.32 \%$, and $58.89 \%$, respectively, in the general public, IVF couples, and ART practitioners. Most participants agreed that the psychological well-being of offspring should be prior considered before making a PAR decision ( $81.84 \%, 73.61 \%$, and $76.98 \%$, respectively). Multivariable logistic regression analysis showed that age, marital status, and gender were common influencing factors while occupation, religion, and pregnancy history showed no support. Males and females showed similar predictive abilities for his/her partner's attitudes toward PAR ( $57.87 \%$ for males, $61.12 \%$ for females). Intercouple agreement analysis showed that the consistent rate was $65.28 \%$ in the attitude toward PAR.

\section{Conclusion}

The findings suggested that the approval rate toward PAR was relatively high in China. Legislation and ethics guidelines for PAR maybe be considered in China. The psychological well-being of offspring should be considered before the PAR execution. Due to the huge regional and population differences in China, investigation of larger participants is necessary.

\section{Introduction}

In recent years, assisted reproductive technology has become a usual and effective treatment for couples with infertility. With the advanced embryo culture technology and increasing pregnancy rate PR, more and more embryos are frozen in reproductive centers. For embryo cryopreservation, ethical and legal problems have emerged. How to deal with the remaining frozen embryos becomes complicated and challenging when a family structure changes (such as accidental death). Therefore, posthumous assisted reproduction (PAR) was chosen as the main topic. 
PAR refers to using gametes or embryos to initiate conception after the death of a genetic parent[1, 2], which brings out a lot of controversial problems. Global countries have different regulatory frameworks for posthumous reproduction. The complex ethical and legal issues result in numerous countries-Canada, France, Germany, Norway, and Sweden-banning the procedure[3, 4]. However, certain countries such as America[5], Australia[6], and Israel[7] either allow it with some limitations or do not regulate it. Both the American Society for Reproductive Medicine (ASRM) and the European Society for Human Reproduction and Embryology (ESHRE) have discussed posthumous reproduction repeatedly. From 2004 to 2018, ASRM has updated three editions[5, 8, 9] regarding PAR's related issues. In 2006, ESHRE Task Force analyzed the ethical aspects of PAR and concluded that a partner's posthumous reproduction was acceptable if the deceased person had given a written consent, and a one-year minimum waiting period was imposed before a PAR treatment[10].

However, there is no relevant research and multi-dimensional study on the reproductive problems of Chinese people. More prominently, legislation and ethics guidelines are lacking. According to the Chinese Health Ministry's administrative decree, all reproductive centers must not provide any assisted reproductive treatment (ART) to single women, and surrogacy is forbidden[11]. Therefore, when facing PAR, concerned families have to destroy the rest frozen embryos, making the patients feel unacceptable and reproductive doctors feel so pitiful. In a research of attitudes towards the donation of frozen embryos in Chinese IVF patients, which suggested that the couples have negative willingness to donate or destroy embryos due to the emotional bonds with the embryos[12]. This manuscript seeks to highlight some core issues causing a clinical dilemma for reproductive doctors. The controversial problems are as follows: I) What are the attitudes of the general public, IVF couples, and ART practitioners toward PAR? II) What are the legal attributes of gametes and embryos? III) Who can dispose of frozen embryos or gametes in the hospital when one of the members of the couple dies? IV) Which should be considered first, family inheritance or offspring' healthy grow-up? V) Can the will of the living spouse represent the will of the deceased?

Based on the above-mentioned problems, this study was designed to measure the attitudes of the general public, IVF couples, and ART practitioners toward PAR and provide more reasonable recommendations to improve frozen embryos' management.

\section{Materials And Methods}

\section{Ethical approval}

was granted by the Ethics Committee of the First Affiliated Hospital of Shantou University Medical College (Approval No. SUMC-ER-R 2020009). Every participant was informed the project purpose and read the Instructions for Participants (Supplemental file 1). Participation was voluntary, and participants were allowed to discontinue participation at any time. Only fully completed surveys were received and analyzed. This assisted in avoiding data deletion.

\section{Questionnaire design}


The flow chart was shown in Figure 1. Three kinds of questionnaires, all consisting of a brief introduction for the participants, followed by 2-5 sections of questions, were distributed to measure the attitudes of the general public, IVF couples, and ART practitioners to PAR.

The first questionnaire was designed for the general public, consisting of four sections. The first section contained seven questions about the participants' personal information to identify the individual influencing factors of the participants' attitudes. The second section, including questions 8-12, mainly investigated the participants' marriage and childbearing information, clarifying the attitudes and decision-making among groups with different pregnancy histories. Three questions were in the third section to investigates the reproductive viewpoints, thus clarifying whether traditional Chinese viewpoints have changed. The last ten questions in the fourth section aimed to examine their attitudes to PAR. Details of the first questionnaire were attached as Supplemental file 2 (also available online: https://www.wjx.cn/jq/50100301.aspx ).

The second questionnaire was divided into five sections for the IVF couples. In addition to the above four same sections in the first questionnaire, another section was added to investigate the consistency of attitudes between husband and wife in decision-making and the mutual prediction of decisions made by the spouses. Details of the second questionnaire were attached as Supplemental file 3 (also available online: https://www.wjx.cn/jq/50099657.aspx ).

The third questionnaire was designed for ART practitioners, including personal information, marriage and childbearing, and basic attitudes. Details of the third questionnaire were attached as Supplemental file 4 (also available online: https://www.wjx.cn/jq/49663627.aspx ).

\section{Study protocol}

Electronic questionnaires (e-questionnaire) were used in all the three survey, produced by the Wenjuanxing Survey System (https://www.wjx.cn), a professional online survey platform. An QR code was then generated and distributed via the social media called Wechat[13], the most frequently used social media in China. WeChat has over 1.24 billion users, $78 \%$ of people in China aged 16-64 are using WeChat[14]. Therefore, questionnaires collected through WeChat would potentially reduce the sample bias and increase the representation of participants.

In the survey of the general public, adult Chinese people with healthy mentality were the target population. Online survey were used. Individuals aged 18 years and older were recruited online. There were no minimum on education levels, monthly incomes and marital status. To make the collected subjects more representative, we chose to distribute the questionnaire in colleges, hospitals, government departments, supermarkets, communities, rural areas, and towns. Participants from different cities were recruited by friend groups in WeChat.

The survey of the IVF couples was conducted in the Reproductive Center, the First Affiliated Hospital of Shantou University Medical College. Infertility couples receiving IVF treatment were the only target population. Patients with ovulation induction or intrauterine insemination were excluded. Patients who intended to receive the IVF treatment but had not yet entered the treatment cycle were not included in the study. The survey was completed when the couples visit doctors simultaneously in person. Only the face-to-face survey was used to 
identify the attitudes of IVF couples, with the interactive prediction within couples being one of the most important results. Our researchers stood beside the couples to prevent them communicating each other. Detailedly, a QR code was provided for the couples to finish the e-questionnaire.

For the attitudes of nationwide ART staff, most questionnaires were handed out during the 13th Annual Conference of the Chinese Society of Reproductive Medicine (CSRM). Some questionnaires were recruited by friend groups in WeChat. Male-specialist, female-specialist, nurse, and embryologists were the target population. There were no limits on marital status, pregnancy history, and professional level.

\section{Analysis}

Data were analyzed by IBM SPSS Statistics (Version 22.0). The descriptive statistical analysis was applied to compare general demographic characteristics. Then the Chi-square test was utilized to compare the intergroup differences, followed by multivariable logistic regression analysis for the influencing factors of PAR. An inter-rater agreement statistic was used in the study of predicting the accuracy of attitudes between spouses. The kappa index was evaluated. All reported $P$ values were two-sided, and $P<0.05$ was considered significant.

\section{Results}

\section{General demographic data}

This study was conducted from November 2019 to February 2021. As shown in Figure 1, 2181 members of the public completed the first questionnaire. Of those, 2072 participants were included for the analysis (response rate: 95\%). 109 subjects were excluded due to age. 432 IVF patients and 304 ART practitioners were included in questionnaires 2 and 3 with no deleted data. The demographic characteristics of participants were shown in Table 1. The survey was delayed for few months because of the nCoV-2019 outbreak. The demographic characteristics of the participants in each group were shown in Table 1. Models were created to analyze demographic characteristics, including gender, age, educational level, occupation, monthly income, religion, household registration, marital status, pregnancy history, and child numbers. Additionally, some adjustments were applied according to different populations. For example, infertility years and professional levels were analyzed only in IVF couples or ART practitioners. As a result, the mean ages were $30.77 \pm 8.69$ (aged 18 - 68) in the general public, $32.83 \pm 4.63$ (aged 23 - 47) in IVF couples, and $34.08 \pm 8.09$ (aged 20 - 56) in ART practitioners. More than half (58.96\%) of the general public and $63.48 \%$ of ART practitioners within the subjects were married. The IP addresses of the participants covered more than 30 cities of 18 provinces in public (Supplemental Figure 1) and 21 provinces in IVF practitioners (Supplemental Figure 2). The sufficient and appropriate proportion of married reproductive-aged participants indicated that the participants' primary demographic data could ensure the validity of this study. 
Table 1

Demographic characteristics of participants.

\begin{tabular}{|c|c|c|c|c|c|}
\hline \multicolumn{2}{|c|}{ General public $(\mathrm{N}=2076)$} & \multicolumn{2}{|c|}{ IVF patients $(\mathrm{N}=432)$} & \multicolumn{2}{|c|}{ ART practitioners $(\mathrm{N}=304)$} \\
\hline items & $N(\%)$ & items & $\mathrm{N}(\%)$ & items & $N(\%)$ \\
\hline Gender & & Age $(y)$ & & Age $(y)$ & \\
\hline Male & $644(31.02 \%)$ & $23-34$ & $280(64.81 \%)$ & $20-29$ & $96(31.59 \%)$ \\
\hline Female & $1432(68.98 \%)$ & $35-44$ & $152(35.19 \%)$ & $30-39$ & $131(43.09 \%)$ \\
\hline $\operatorname{Age}(y)$ & & Education & & $40-49$ & $62(20.39 \%)$ \\
\hline $18-24$ & $509(24.52 \%)$ & $\begin{array}{l}\text { Below } \\
\text { college }\end{array}$ & $188(43.52 \%)$ & $\geq 50$ & $15(4.93 \%)$ \\
\hline $25-34$ & 1058(50.96\%) & College & $182(42.13 \%)$ & Education & \\
\hline $35-44$ & 309 (14.88\%) & $\begin{array}{l}\text { Post- } \\
\text { graduate }\end{array}$ & $62(14.35 \%)$ & Bachelor\&Below & $139(45.72 \%)$ \\
\hline$\geq 45$ & $196(9.44 \%)$ & Occupation & & Master degree & $126(41.45 \%)$ \\
\hline Education & & $\begin{array}{l}\text { Liberal } \\
\text { work }\end{array}$ & $51(11.81 \%)$ & PHD degree & $39(12.83 \%)$ \\
\hline $\begin{array}{l}\text { Below } \\
\text { college }\end{array}$ & $270(13.01 \%)$ & business & $138(31.94 \%)$ & Occupation & \\
\hline College & $1300(62.62 \%)$ & $\begin{array}{l}\text { General } \\
\text { staff }\end{array}$ & $162(37.50 \%)$ & Male-specialist & $36(11.84 \%)$ \\
\hline $\begin{array}{l}\text { Post- } \\
\text { graduate }\end{array}$ & $506(24.37 \%)$ & $\begin{array}{l}\text { Technical } \\
\text { post }\end{array}$ & $81(18.75 \%)$ & $\begin{array}{l}\text { Female- } \\
\text { specialist }\end{array}$ & $107(35.20 \%)$ \\
\hline Occupation & & $\begin{array}{l}\text { Monthly } \\
\text { income (¥) }\end{array}$ & & Nurse & $91(29.93 \%)$ \\
\hline $\begin{array}{l}\text { Liberal } \\
\text { work }\end{array}$ & $367(17.68 \%)$ & $\leq 3000$ & $99(22.92 \%)$ & Lab-technicians & $70(23.03 \%)$ \\
\hline business & $120(5.78 \%)$ & $3001-6000$ & $153(35.42 \%)$ & $\begin{array}{l}\text { Professional } \\
\text { level }\end{array}$ & \\
\hline $\begin{array}{l}\text { General } \\
\text { staff }\end{array}$ & $414(19.94 \%)$ & $6001-9000$ & $82(18.98 \%)$ & Primary & 95 (31.25\%) \\
\hline $\begin{array}{l}\text { Technical } \\
\text { post }\end{array}$ & $1175(56.60 \%)$ & $\geq 9001$ & $98(22.68 \%)$ & Secondary & $112(36.84 \%)$ \\
\hline $\begin{array}{l}\text { Monthly } \\
\text { income (¥) }\end{array}$ & & Religion & & Vice-senior & 64 (21.05\%) \\
\hline$\leq 3000$ & 494 (23.80\%) & Buddhism & $120(27.78 \%)$ & Senior & $33(10.86 \%)$ \\
\hline $3001-6000$ & 485 (23.36\%) & Christian & $4(0.93 \%)$ & Marital status & \\
\hline 6001-9000 & 455 (21.92\%) & Others & $10(2.31 \%)$ & married & 193(63.49\%) \\
\hline
\end{tabular}




\begin{tabular}{|c|c|c|c|c|c|}
\hline \multicolumn{2}{|c|}{ General public $(\mathrm{N}=\mathbf{2 0 7 6})$} & \multicolumn{2}{|c|}{ IVF patients $(\mathrm{N}=432)$} & \multicolumn{2}{|c|}{ ART practitioners $(\mathrm{N}=304)$} \\
\hline$\geq 9001$ & $642(30.92 \%)$ & No & $298(68.98 \%)$ & Single & $111(35.51 \%)$ \\
\hline Religion & & Registration & & $\begin{array}{l}\text { Pregnancy } \\
\text { history }\end{array}$ & \\
\hline Buddhism & $299(14.40 \%)$ & Rural & $272(62.961 \%)$ & Yes & $162(53.29 \%)$ \\
\hline Christian & $48(2.31 \%)$ & Urban & $160(37.04 \%)$ & NO & $142(46.71 \%)$ \\
\hline Others & $65(3.13 \%)$ & $\begin{array}{l}\text { Marital } \\
\text { status }\end{array}$ & & $\begin{array}{l}\text { Conceived } \\
\text { manner }\end{array}$ & $(\mathrm{N}=162)$ \\
\hline No & $1664(80.15 \%)$ & First & $375(86.81 \%)$ & Nature & $130(80.25 \%)$ \\
\hline Registration & & remarried & $57(13.19 \%)$ & ART & $32(19.75 \%)$ \\
\hline Rural & 757 (36.45\%) & $\begin{array}{l}\text { Years of } \\
\text { infertile }\end{array}$ & & Children & $(\mathrm{N}=162)$ \\
\hline Urban & $1319(63.54 \%)$ & $\leq 1$ & 27 (6.25\%) & 1 & $86(53.09 \%)$ \\
\hline $\begin{array}{l}\text { Marital } \\
\text { status }\end{array}$ & & $1-4$ & $199(46.07 \%)$ & $\geq 2$ & $55(33.95 \%)$ \\
\hline married & $1224(58.96 \%)$ & $4-7$ & $156(36.11 \%)$ & None & $21(12.96 \%)$ \\
\hline Single & $852(41.04 \%)$ & $\geq 7$ & $50(11.57 \%)$ & & \\
\hline $\begin{array}{l}\text { Marriage } \\
\text { (y) }\end{array}$ & $(\mathrm{N}=1224)$ & $\begin{array}{l}\text { Pregnancy } \\
\text { history }\end{array}$ & & & \\
\hline$\leq 1$ & $106(8.66 \%)$ & Yes & $196(45.37 \%)$ & & \\
\hline $2-4$ & $275(22.47 \%)$ & No & $236(54.63 \%)$ & & \\
\hline $4-7$ & $267(21.81 \%)$ & $\begin{array}{l}\text { Conceived } \\
\text { manner }\end{array}$ & $(\mathrm{N}=196)$ & & \\
\hline$\geq 7$ & $576(47.06 \%)$ & Nature & $106(54.08 \%)$ & & \\
\hline $\begin{array}{l}\text { Pregnancy } \\
\text { history }\end{array}$ & & ART & $90(45.92 \%)$ & & \\
\hline Yes & $1067(51.40 \%)$ & Children & $(\mathrm{N}=196)$ & & \\
\hline NO & $1009(48.60 \%)$ & 1 & 83 (42.35\%) & & \\
\hline $\begin{array}{l}\text { Conceived } \\
\text { manner }\end{array}$ & $(\mathrm{N}=1067)$ & $\geq 2$ & 18 (9.18\%) & & \\
\hline Nature & $1040(97.47 \%)$ & None & 95 (48.47\%) & & \\
\hline ART & 27 (2.53\%) & & & & \\
\hline Children & $(\mathrm{N}=1224)$ & & & & \\
\hline 1 & 546 (44.61\%) & & & & \\
\hline
\end{tabular}




\begin{tabular}{|lll|}
\hline General public $(\mathrm{N}=\mathbf{2 0 7 6})$ & IVF patients $(\mathrm{N}=\mathbf{4 3 2})$ & ART practitioners $(\mathrm{N}=\mathbf{3 0 4})$ \\
\hline$\geq 2$ & $378(30.88 \%)$ & \\
\hline None & $300(24.51 \%)$ & \\
\hline
\end{tabular}

\section{Traditional Reproductive Viewpoints}

The traditional reproductive attitudes were investigated in the general public and IVF couples (Figure 2). Within the included subjects, over half $(52.46 \%)$ of the general public believed that children were essential for a family. This attitude was more assertive in IVF couples (89.58\%). Surprisingly, 1753 of 2076 public (84.44\%) believed that not only boys could carry on the family line, which was significantly lower in IVF patients $(51.39 \%)$. More than half $(53.81 \%)$ of the public and $43.75 \%$ of IVF patients believed that adopted children can also carry on the family line. Significant discordance regarding whether adopted children can also carry on the family line $\left(X^{2}=35.11, P=0.000\right)$ existed between the general public and the IVF couples.

\section{Attitudes Toward Par Related Issues}

The attitudes of the general public, IVF patients, and ART practitioners toward PAR-related issues were shown in Figure 3. All the three groups showed high approval rates for PAR when the deceased's will is clear $(79.10 \%$, $55.32 \%$, and $58.89 \%$, respectively). Within the included subjects, only part of IVF patients (42.59\%) and ART practitioners $(28.95 \%)$ still support PAR when the willingness of the deceased is unclear, which is significantly different from the opinion in general public $(72.59 \%, P=0.000)$. Roughly half of the public participants $(53.23 \%)$ and IVF couples (44.68\%) thought it unnecessary to allow adequate time for grieving and insisted that the spouse alive has the right to choose to transplant the posthumous embryos. However, 168 of 304 ART practitioners (59.26\%) thought it necessary to allow adequate grieving time. When asked "how long is suitable for grieving", more IVF couples (62.27\%) tended to prefer a shorter grieving time (for 0-1 year) than the general public (34.10\%) and ART practitioners (28.29\%). Unsurprisingly, attitudes of the three groups on the growth of offspring's mental health were highly consistent ( $81.84 \%$ for general public, $73.61 \%$ for IVF couples, and $76.98 \%$ for ART practitioners).

\section{Influencing Factors Toward Par-related Issues}

Multivariable logistic regression analysis was applied to identify PAR's influencing factors in the three groups (Table 2). Within the included subjects, the demographic characteristics associated with support for PAR were different in the three groups. However, age, marital status, and gender were common influencing factors. In the general public group, multivariable logistic regression analysis showed that younger (OR 3.48 for below 34 years old), married (OR $3.46,95 \% \mathrm{Cl} 1.28-9.36$ ), and lower-income participants (OR 2.53 for monthly income below $3000 ¥$ and OR 1.75 for 3001-6000 $¥$ ) had a positive relationship with supporting PAR. However, a negative significance was found in male participants (OR 0.54, 95\% $\mathrm{Cl} 0.38-0.77$ ) and the lower educated participants (OR $0.04,95 \% \mathrm{Cl} 0.02-0.07$ for those below college education). No significant associations were 
found in occupation, religion, household registration, pregnancy history, and child numbers. In the IVF patients, a similar positive significance was found in younger (OR 4.54 for below 34 years old), first married patients (OR 5.07, 95\% $\mathrm{Cl} 1.96-13.13$ ). A strong negative association appeared in males (OR 0.41, 95\% $\mathrm{Cl} 0.21-0.83$ ), the lower educated (OR $0.05,95 \% \mathrm{Cl}$ 0.01-0.19 for those below college education), and more low-income patients (OR 0.27 for monthly income below $3000 ¥$ and OR 0.33 for 3001-6000 $¥$ ). Occupation, religion, household registration, pregnancy history did not influence the attitudes of PAR. Although the gender of the practitioners was not investigated, the results indicated that female fertility specialists showed a higher supporting rate (OR $2.42,95 \% \mathrm{Cl} 1.05-5.58$ ). As with the results of the front two groups, higher supportive attitudes were found in younger and married practitioners (OR 4.83 and 3.80 , respectively). IVF practitioners having a conceived history showed a stronger support (OR 7.18, 95\% $\mathrm{Cl} 1.67-31.12$ ) which was different with the results of the previous two groups. 
Table 2

Logistic regression predicting demographic characteristics associated with supporting PAR.

\begin{tabular}{|c|c|c|c|c|c|c|c|c|c|}
\hline & \multicolumn{3}{|c|}{ Public } & \multicolumn{3}{|c|}{ IVF couples } & \multicolumn{3}{|c|}{ ART practitioners } \\
\hline & $\mathbf{P}$ & OR & $95 \% \mathrm{Cl}$ & $\mathbf{P}$ & OR & $95 \% \mathrm{Cl}$ & $\mathbf{P}$ & OR & $95 \% \mathrm{Cl}$ \\
\hline \multicolumn{7}{|l|}{ Gender } & \multirow{3}{*}{\multicolumn{3}{|c|}{ Not investigated }} \\
\hline Male & $0.00^{*}$ & 0.54 & $\begin{array}{l}0.38- \\
0.77\end{array}$ & $0.01^{*}$ & 0.41 & $\begin{array}{l}0.21- \\
0.83\end{array}$ & & & \\
\hline Female & \multicolumn{3}{|c|}{ Referent } & \multicolumn{3}{|c|}{ Referent } & & & \\
\hline \multicolumn{10}{|l|}{ Age group } \\
\hline$\leq 34$ & $0.00^{*}$ & 3.48 & $\begin{array}{l}1.76- \\
6.90\end{array}$ & $0.01^{*}$ & 4.54 & $\begin{array}{l}1.53- \\
13.56\end{array}$ & $0.01^{*}$ & 4.83 & $\begin{array}{l}1.55- \\
15.07\end{array}$ \\
\hline$\geq 35$ & \multicolumn{3}{|c|}{ Referent } & \multicolumn{3}{|c|}{ Referent } & \multicolumn{3}{|c|}{ Referent } \\
\hline \multicolumn{7}{|l|}{ Education level } & \multicolumn{3}{|c|}{ Education Level a } \\
\hline Below college & $0.00^{*}$ & 0.04 & $\begin{array}{l}0.02- \\
0.07\end{array}$ & $0.00^{*}$ & 0.05 & $\begin{array}{l}0.01- \\
0.19\end{array}$ & 0.66 & 1.27 & $\begin{array}{l}0.44- \\
3.61\end{array}$ \\
\hline College degree & 0.34 & 0.79 & $\begin{array}{l}0.48- \\
1.29\end{array}$ & 0.07 & 0.28 & $\begin{array}{l}0.07- \\
1.10\end{array}$ & $0.03^{*}$ & 3.18 & $\begin{array}{l}1.10- \\
9.16\end{array}$ \\
\hline Above college & \multicolumn{3}{|c|}{ Referent } & \multicolumn{3}{|c|}{ Referent } & \multicolumn{3}{|c|}{ Referent } \\
\hline \multicolumn{7}{|l|}{ Occupation } & \multicolumn{3}{|c|}{ Occupation ${ }^{b}$} \\
\hline Liberal work & 0.11 & 0.58 & $\begin{array}{l}0.29- \\
1.14\end{array}$ & 0.07 & 3.12 & $\begin{array}{l}0.91- \\
10.72\end{array}$ & 0.18 & 2.06 & $\begin{array}{l}0.71- \\
5.94\end{array}$ \\
\hline Business & 0.92 & 1.03 & $\begin{array}{l}0.54- \\
1.97\end{array}$ & 0.24 & 1.75 & $\begin{array}{l}0.68- \\
4.45\end{array}$ & $0.04^{*}$ & 2.42 & $\begin{array}{l}1.05- \\
5.58\end{array}$ \\
\hline General staff & 0.69 & 1.11 & $\begin{array}{l}0.68- \\
1.79\end{array}$ & 0.22 & 1.89 & $\begin{array}{l}0.69- \\
5.12\end{array}$ & 0.08 & 2.22 & $\begin{array}{l}0.91- \\
5.39\end{array}$ \\
\hline
\end{tabular}

Note:

a Education level in ART practitioners was classified into "Below bachelor", "Master", and "PhD". PhD was set as the referent.

b Occupation status in ART practitioners was classified into "Male-fertility specialist", "Female-fertility specialist", "Laboratory technicians" and "Nurse". Nurse was set as the referent.

c Professional level in ART practitioners was classified into "Primary", "Secondary", "Vice-senior", and "Senior". Senior was set as the referent.

d Marital status in IVF couples was classified into "First married", and "Remarried". Remarried was set as the referent.

* Significant items, P < 0.05; Cl: Confidence Interval; OR: Odds Ratio. 


\begin{tabular}{|c|c|c|c|c|c|c|c|c|c|}
\hline & \multicolumn{3}{|l|}{ Public } & \multicolumn{3}{|c|}{ IVF couples } & \multicolumn{3}{|c|}{ ART practitioners } \\
\hline Technical post & \multicolumn{3}{|c|}{ Referent } & \multicolumn{3}{|c|}{ Referent } & \multicolumn{3}{|c|}{ Referent } \\
\hline \multicolumn{7}{|l|}{$\begin{array}{l}\text { Monthly income } \\
(¥)\end{array}$} & \multicolumn{3}{|c|}{ Professional level ${ }^{c}$} \\
\hline$\leq 3000$ & $0.01^{*}$ & 2.53 & $\begin{array}{l}1.28- \\
5.03\end{array}$ & $0.04^{*}$ & 0.27 & $\begin{array}{l}0.08- \\
0.94\end{array}$ & 0.31 & 0.43 & $\begin{array}{l}0.08- \\
2.20\end{array}$ \\
\hline $3001-6000$ & $0.03^{*}$ & 1.75 & $\begin{array}{l}1.05- \\
2.93\end{array}$ & $0.03^{*}$ & 0.33 & $\begin{array}{l}0.13- \\
0.87\end{array}$ & 0.07 & 0.27 & $\begin{array}{l}0.07- \\
1.12\end{array}$ \\
\hline $6001-9000$ & 0.21 & 1.37 & $\begin{array}{l}0.84- \\
2.25\end{array}$ & 0.35 & 0.58 & $\begin{array}{l}0.19- \\
1.82\end{array}$ & 0.13 & 0.34 & $\begin{array}{l}0.08- \\
1.39\end{array}$ \\
\hline$\geq 9001$ & \multicolumn{3}{|c|}{ Referent } & \multicolumn{3}{|c|}{ Referent } & \multicolumn{3}{|c|}{ Referent } \\
\hline \multicolumn{7}{|l|}{ Religion } & \multirow{2}{*}{\multicolumn{3}{|c|}{ Not investigated }} \\
\hline Yes & 0.06 & 1.53 & $\begin{array}{l}0.98- \\
2.39\end{array}$ & 0.09 & 1.92 & $\begin{array}{l}0.91- \\
4.04\end{array}$ & & & \\
\hline No & \multicolumn{3}{|c|}{ Referent } & \multicolumn{3}{|c|}{ Referent } & & & \\
\hline \multicolumn{7}{|l|}{$\begin{array}{l}\text { House } \\
\text { registration }\end{array}$} & \multirow{3}{*}{\multicolumn{3}{|c|}{ Not investigated }} \\
\hline Rural & 0.64 & 1.10 & $\begin{array}{l}0.74- \\
1.62\end{array}$ & 0.07 & 1.98 & $\begin{array}{l}0.94- \\
4.17\end{array}$ & & & \\
\hline Urban & \multicolumn{3}{|c|}{ Referent } & \multicolumn{3}{|c|}{ Referent } & & & \\
\hline \multicolumn{4}{|l|}{ Marital status } & \multicolumn{3}{|c|}{ Marital status $^{\mathrm{d}}$} & & & \\
\hline married & $0.01^{*}$ & 3.46 & $\begin{array}{l}1.28- \\
9.36\end{array}$ & $0.00^{*}$ & 5.07 & $\begin{array}{l}1.96- \\
13.13\end{array}$ & $0.01^{*}$ & 3.80 & $\begin{array}{l}1.33- \\
10.83\end{array}$ \\
\hline Single & \multicolumn{3}{|c|}{ Referent } & \multicolumn{3}{|c|}{ Referent } & \multicolumn{3}{|c|}{ Referent } \\
\hline \multicolumn{10}{|l|}{$\begin{array}{l}\text { Pregnancy } \\
\text { history }\end{array}$} \\
\hline \multicolumn{10}{|l|}{ Note: } \\
\hline \multicolumn{10}{|c|}{$\begin{array}{l}\text { a Education level in ART practitioners was classified into "Below bachelor", "Master", and "PhD". PhD was } \\
\text { set as the referent. }\end{array}$} \\
\hline \multicolumn{10}{|c|}{$\begin{array}{l}\text { b Occupation status in ART practitioners was classified into "Male-fertility specialist", "Female-fertility } \\
\text { specialist", "Laboratory technicians" and "Nurse". Nurse was set as the referent. }\end{array}$} \\
\hline \multicolumn{10}{|c|}{$\begin{array}{l}\text { "Professional level in ART practitioners was classified into "Primary", "Secondary", "Vice-senior", and } \\
\text { "Senior". Senior was set as the referent. }\end{array}$} \\
\hline \multicolumn{10}{|c|}{$\begin{array}{l}\text { d Marital status in IVF couples was classified into "First married", and "Remarried". Remarried was set as } \\
\text { the referent. }\end{array}$} \\
\hline
\end{tabular}




\begin{tabular}{|c|c|c|c|c|c|c|c|c|c|}
\hline \multirow{2}{*}{ Yes } & \multicolumn{3}{|c|}{ Public } & \multicolumn{3}{|c|}{ IVF couples } & \multicolumn{3}{|c|}{ ART practitioners } \\
\hline & 0.41 & 0.56 & $\begin{array}{l}0.14- \\
2.23\end{array}$ & 0.56 & 0.70 & $\begin{array}{l}0.21- \\
2.30\end{array}$ & $0.01^{*}$ & 7.18 & $\begin{array}{l}1.67- \\
31.12\end{array}$ \\
\hline NO & \multicolumn{3}{|c|}{ Referent } & \multicolumn{3}{|c|}{ Referent } & \multicolumn{3}{|c|}{ Referent } \\
\hline \multicolumn{10}{|c|}{$\begin{array}{l}\text { Conceived } \\
\text { manner }\end{array}$} \\
\hline Nature & $0.03^{*}$ & 3.71 & $\begin{array}{l}1.14- \\
12.07\end{array}$ & 0.81 & 0.87 & $\begin{array}{l}0.28- \\
2.73\end{array}$ & 0.66 & 0.76 & $\begin{array}{l}0.23- \\
2.77\end{array}$ \\
\hline ART & \multicolumn{3}{|c|}{ Referent } & \multicolumn{3}{|c|}{ Referent } & \multicolumn{3}{|c|}{ Referent } \\
\hline \multicolumn{10}{|c|}{ Children } \\
\hline 1 & 0.49 & 0.81 & $0.44-$ & 0.96 & 1.03 & $\begin{array}{l}0.35- \\
3.00\end{array}$ & 0.78 & 1.17 & $\begin{array}{l}0.40- \\
3.36\end{array}$ \\
\hline$\geq 2$ & 0.16 & 1.63 & $\begin{array}{l}0.83- \\
3.22\end{array}$ & 0.62 & 2.06 & $\begin{array}{l}0.12- \\
36.00\end{array}$ & 0.85 & 0.90 & $\begin{array}{l}0.29- \\
2.77\end{array}$ \\
\hline None & \multicolumn{3}{|c|}{ Referent } & \multicolumn{3}{|c|}{ Referent } & \multicolumn{3}{|c|}{ Referent } \\
\hline \multicolumn{10}{|l|}{ Note: } \\
\hline \multicolumn{10}{|c|}{$\begin{array}{l}\text { a Education level in ART practitioners was classified into "Below bachelor", "Master", and "PhD". PhD was } \\
\text { set as the referent. }\end{array}$} \\
\hline \multicolumn{10}{|c|}{$\begin{array}{l}\text { b Occupation status in ART practitioners was classified into "Male-fertility specialist", "Female-fertility } \\
\text { specialist", "Laboratory technicians" and "Nurse". Nurse was set as the referent. }\end{array}$} \\
\hline \multicolumn{10}{|c|}{$\begin{array}{l}\text { "Professional level in ART practitioners was classified into "Primary", "Secondary", "Vice-senior", and } \\
\text { "Senior". Senior was set as the referent. }\end{array}$} \\
\hline \multicolumn{10}{|c|}{$\begin{array}{l}\text { d Marital status in IVF couples was classified into "First married", and "Remarried". Remarried was set as } \\
\text { the referent. }\end{array}$} \\
\hline
\end{tabular}

\section{The intercouple agreement and ability in predicting their spouse preferences toward PAR}

Whether the attitudes of the living spouses could represent the actual willingness of the deceased was explored by comparing the prediction by husbands/wives with actual choices of their wives/husbands. Within the included subjects, the prediction accuracy of husbands and wives was $57.87 \%(125 / 216$, Table 3$)$ and $61.12 \%(132 / 216$, Table 4), respectively. The inter-rater agreement analysis showed that unsatisfied Kappa values were found both in the prediction accuracy of husbands (Kappa value: 0.338 ) and wives (Kappa value: 0.408). Over half $(112 / 216,51.85 \%$, Table 5) wives showed approvals for PAR, while the rate was lower in husbands ( $39.81 \%, X^{2}=7.09, P=0.03$, Table 5). Intercouple agreement analysis shows that the agreement rate within couples was $65.28 \%$ (Table 5). When the participants were asked "whether the attitudes are consistent in important decision-makings within couples (Question 17 in the second questionnaire)", 243 of 
432 participants $(56.25 \%)$ agreed that their decisions were always the same, a little lower than the actual prediction rate of males.

Table 3

Accuracy of males in predicting their spouse preference for PAR

\begin{tabular}{|llll|}
\hline \multicolumn{4}{|c|}{ Male's prediction ${ }^{\circ}$} \\
\hline Female's attitude & Destroy & Donate for research & For PAR \\
\hline Destroy & $43(19.90 \%)$ & $2(0.93 \%)$ & $13(6.02 \%)$ \\
\hline Donate for research & $12(5.55 \%)$ & $19(8.80 \%)$ & $15(6.94 \%)$ \\
\hline For PAR & $30(13.89 \%)$ & $19(8.80 \%)$ & $63(29.17 \%)$ \\
\hline Note: & & & \\
\hline $\begin{array}{l}\text { a the accuracy rate of males in predicting their spouse preference for PAR was just } 57.87 \% \text { and the Kappa } \\
\text { index was 0.338. }\end{array}$
\end{tabular}

Table 4

Accuracy of females in predicting their spouse preference for PAR

\begin{tabular}{|llll|}
\hline \multicolumn{4}{c|}{ Female's prediction a } \\
\hline Male's attitude & Destroy & Donate for research & For PAR \\
\hline Destroy & $50(23.15 \%)$ & $8(3.70 \%)$ & $22(10.19 \%)$ \\
\hline Donate for research & $17(7.87 \%)$ & $26(12.04 \%)$ & $7(3.24 \%)$ \\
\hline For PAR & $5(2.31 \%)$ & $25(11.57 \%)$ & $56(25.93 \%)$ \\
\hline Note: & & & \\
\hline $\begin{array}{l}\text { a the accuracy rate of females in predicting their spouse preference for PAR was just } 61.12 \% \text { and the } \\
\text { Kappa index was 0.408. }\end{array}$ & \\
\hline
\end{tabular}

Table 5

Intercouple agreement on actual selections of the posthumous embryos.

\begin{tabular}{|llll|}
\hline & Destroy & Donate for research & For PAR \\
\hline Male's attitude & $80(37.04 \%)$ & $50(23.15 \%)$ & $86(39.81 \%)$ \\
\hline Female's attitude & $58(26.85 \%)$ & $46(21.30 \%)$ & $112(51.85 \%)$ \\
\hline Intercouple agreement ${ }^{a}$ & $43(19.91 \%)$ & $29(13.43 \%)$ & $69(31.94 \%)$ \\
\hline Note: & & \\
\hline a $X^{2}=7.09, P=0.029$, The agreement rate between couples was $65.28 \%$. \\
\hline
\end{tabular}




\section{Discussion}

To our knowledge, this is the first study to assess the attitudes toward PAR in the Chinese population. Compared with published researches[15-17] from other countries or regions, this study has a larger sample size,and it is the most comprehensive assessment. The results suggest that most people within the included subjects approve of PAR in certain situations.

\section{The changes of the traditional Chinese reproductive viewpoint.}

Under the influence of Confucianism, the mainstream of modern Chinese society as one of the most crucial spiritual wealth in the 5000 years' Chinese culture, an old Chinese saying goes, "of three forms of unfilial behaviors, the worst is to have no descendants". Having descendants has been the most critical reproductive viewpoint in China under the influence of Confucianism. Traditional Chinese believe that only a son can carry on the family line. However, the traditional viewpoint has been changed nowadays in China (Figure 2). On the one hand, this change is partially related to the improved educational level in China brought by building the most extensive higher education system globally. The gross enrollment rate of a college education has reached 51.6\%[18], showing that China has entered into the popularization of higher education[19]. participants with higher education showed a more open-mind reproductive viewpoint (Table 2), believing that children are unnecessary, and boys and girls should be equal in intergenerational transmission.

On the other hand, the "one-child policy", promulgated in 1971 by the Chinese government, may be related to the changing in reproductive viewpoints of Chinese people. Each family could have only one child in the past 40 years. This generation of only child has become the main population in current Chinese society with a new reproductive viewpoint influenced by the restrictive family planning policy. Even some families are Double Income No Kids (DINKs). According to the China Fertility Report 2019[20], DINKs households accounted for about $10 \%$ of Shenzhen registration households, and there were 600000 DINKs households with an increasing trend in China in 2010. This research showed that some traditional Chinese reproductive viewpoints have changed over generations with the initiation of national policy, education extension, lifestyle changes, and economic development.

\section{The influencing factors toward PAR attitudes.}

Supporting PAR was positively associated with young participants and married individuals, which was similarly demonstrated in the previous research[16]. However, gender was found to be an influencing factor toward PAR attitudes. Supporting PAR seemed easier for females because women are the main body of pregnancy, having more and stronger feelings about pregnancy and childbirth. Traditional Confucianism strengthened the family identity of females as a fertility tool and their responsibility to bear and rear children. Besides, the difference in PAR acceptance in males and females is due to the complexity of PAR after female partners decease, as male partners need surrogates or another female partner to carry the pregnancy. No significant difference toward education existed in ART practitioners because the lowest education level of ART practitioners was college degrees, which already belonged to the high education group. Education was no longer the core factor affecting people's attitudes to PAR. As for the factor of occupation, a significant difference was only reflected in ART practitioners, but not in the general public and IVF patients. The possible reason is that ART practitioners keep a different degree of contact and emotion with patients or embryos in 
various aspects. Hence, ART practitioners of different identities have different attitudes toward PAR. Female specialist could understand parents' need for fertility and the hard-earned embryos by contacting IVF couples, making them to be more inclined to help patients achieve their longing reproductive needs. The correlations between income levels and PAR attitudes were inconsistent in different populations. The working relationship or income levels may be affected by IVF treatment possibly. Many IVF patients were reduced to working parttime or even resignation due to the treatments, leading to inconsistent results [21,22]. The conceived manner showed a significant difference only in general public because of only 27 participants with an ART-conceived manner were included, accounting for $2.5 \%$ of the participants having pregnancy history. The relatively broad $95 \% \mathrm{Cl}$ value reflects the data bias partly. More participants with an history of ART-conceived manner may reverse this result. Therefore, the demographic characteristics need to be fully considered before implementing PAR.

\section{Willingness of the deceased or his/her spouse, which should be firstly considered?}

The approval rates of the three groups are relatively high when a consent exists (Figure 3). Within the included subjects, $42.59 \%$ of IVF patients approve of their spouses to use the posthumous embryos for PAR with no prior explicit consent, lower than recent similar research [23]. Peoples suggesting the willingness of the living spouses should come first believed that the initial purpose of acquiring embryos was to deliver a child, and the spouses had a firm willingness and desire for a baby. Therefore, it seemed reasonable to allow spouses to use posthumous embryos for PAR unless there was other evidences indicated that the deceased would have opposed it[24]. However, more than $20 \%$ of IVF patients disagreed with using embryos for PAR after death, suggesting the uncertainty of individuals desiring to have children before death does not represent a willingness to have children after death. Besides, attitudes to PAR were not always concordant within IVF couples $(65.28 \%$ consistant rate, Table 5$)$. Therefore, the living spouses requiring to initiate PAR may not always represent the desires of the dead ones. As a result, in a situation without explicit consent before death, PAR is detrimental to the reproductive autonomy of the deceased. The key lies in best exercising the reproductive autonomy of the deceased after death[25] is most important. Therefore, in the situation of no consent, PAR should be more careful to implemented more carefully after weighing the interests and rights of each party without consent[7].

\section{Rights And Healthy Growth Of Offspring}

PAR is artificially creating single-parent children or even orphans[26]. Undoubtedly, these children will face much mental stress even greater than that of traditional single-parent children as they grow up. Questions have arisen about how PAR affects the posthumous offspring emotionally and psychologically[27, 28]. A negative effect is that children will feel wronged or stigmatized when they find themselves conceived after one genetic parent dies[10]. Another concern is that offspring may consider themselves a commemorative child or a replacement of the deceased. Single-parent children miss many classes, having learning disabilities and attention deficit disorders[29, 30]. They have worse physical health and material resources than the children of married parents[29, 31, 32]. Furthermore, the extreme situation of genetic parent death highlights the offspring's legal rights and welfare insecurity. Without a stable, warm and supportive parent-child relationship,

the children get less family care, less welfare, and more social pressure. The inheritance rights of posthumous children are debatable, given that the genetic parent may have had no intention for PAR[2]. Children's 
inheritance may be diluted by children born after PAR, raising problems in inheritance rights[33]. However, the offspring of PAR plays no role in the decision to initiate their creation. Therefore, they seem to deserve equal benefits that any child is entitled to after a parent dies[10]. Adequate psychological counseling and trauma healing before PAR[34] are essential for ensuring a responsible reproduction rather than an impulsive decision. Children should not be born as substitutes for others, whose welfare and legal rights after birth should be identical to those of double-parent children.

\section{Study Limitations}

Some limitations exist in this research. First, the number of IVF couples, ART practitioners, and participants with below-college education were relatively deficient. Second, to ensure authenticity and validity, and prevent communication and discussion between couples, the survey of IVF couples was only conducted in only one reproductive center, resulting in geographical limitations and some bias. Some research bias may be arisen through the recruiting method. A national and wider investigation is necessary. Last, considering the belief of most participants that offspring' growth was more important than the inheritance of the family generations, further researches are expected to be conducted on the physical and mental health of offspring. More psychological and sociological experts are needed to be involved to give more advice to address this issue.

\section{Conclusions}

PAR is a controversial topic involving psychological, ethical, moral, and legal multi-dimensions. This study confirmed that the approval rates toward PAR in the three groups within the included subjects were generally high. The prediction accuracy and the intercouple concordance in couples were moderate. Traditional Chinese attitudes toward reproduction changed essentially. The psychological well-being of offspring should be considered ahead of PAR implementation. A suitable legal policy or specialized guidance in PAR may be considered and published in China. This research provided some advice or shreds of evidence for medical professionals and policymakers toward practice and policymaking in PAR. Due to the huge regional and population differences in China, investigations in larger participants are required.

\section{List Of Abbreviations}

\section{PAR}

posthumous assisted reproduction

ASRM

the American Society for Reproductive Medicine

ESHRE

the European Society for Human Reproduction and Embryology

ART

assisted reproductive treatment

CSRM

the Chinese Society of Reproductive Medicine

DINKs

Double Income No Kids 


\section{Declarations}

Code availability: Not applicable.

Ethics approval: This study was granted by the Ethics Committee of the First Affiliated Hospital of Shantou University Medical College (Approval No. SUMC-ER-R 2020009).

Consent to participate: Every participant was informed the project purpose and read the Instructions for Participants. Participation was voluntary, and participants were allowed to discontinue participation at any time.

Consent for publication: Not applicable.

Availability of data and material: Original data can be available from corresponding author when needed.

Conflicts of interests: All authors declare no conflict of interests in this study.

Funding: This study was supported by the National Science Foundation of China (No. 81871223, No. 81671536 and No. 81471522).

Authors' contributions: Jiliang Huang: Conceptualization, Manuscript Writing, and Investigation. Jue Li: Formal Analysis, and Investigation. Zhiling Li: Project Administration, and Funding Acquisition. Wanfen Xiao: Project Administration and Revise the manuscript.

\section{Acknowledgements}

Firstly, thanks IPSEN China and CSRM for organizing the IVF New-hot Speech Competition, which let me conceived this idea to do this survey. We also thank the 13th Annual Conference of the Chinese Society of Reproductive Medicine (CSRM) for providing a platform to collect attitudes of national reproductive practitioners. Thanks Dr. Shaojie Pang for the guidance in statistics. Finally, thanks all the participants who joined in this survey.

\section{References}

1. Nakhuda GS: Posthumous assisted reproduction. Semin Reprod Med 2010, 28:329-335.

2. Nakhuda GS, Wang JG, Sauer MV: Posthumous assisted reproduction: a survey of attitudes of couples seeking fertility treatment and the degree of agreement between intimate partners. Fertility and Sterility 2011, 96:1463-1466.e1461.

3. Bahadur G: Posthumous assisted reproduction. Posthumous assisted reproduction (PAR): cancer patients, potential cases, counselling and consent. Hum Reprod 1996, 11:2573-2575.

4. Lansac J: French law concerning medically-assisted reproduction. Hum Reprod 1996, 11:1843-1847.

5. Medicine ECotASfR: Posthumous retrieval and use of gametes or embryos: an Ethics Committee opinion. Fertil Steril 2018, 110:45-49. 
6. Kroon B, Kroon F, Holt S, Wong B, Yazdani A: Post-mortem sperm retrieval in Australasia. Australian and New Zealand Journal of Obstetrics and Gynaecology 2012, 52:487-490.

7. Ram-Tiktin E, Gilbar R, Fruchter RB, Ben-Ami I, Friedler S, Shalom-Paz E: Expanding the use of posthumous assisted reproduction technique: Should the deceased's parents be allowed to use his sperm? Clinical Ethics 2018, 14:18-25.

8. Medicine ECotASfR: Posthumous reproduction. Fertil Steril 2004, 82 Suppl 1:S260-262.

9. Medicine ECotASfR: Posthumous collection and use of reproductive tissue: a committee opinion. Fertil Steril 2013, 99:1842-1845.

10. Pennings G, de Wert G, Shenfield F, Cohen J, Devroey P, Tarlatzis B: ESHRE Task Force on Ethics and Law 11: Posthumous assisted reproduction. Hum Reprod 2006, 21:3050-3053.

11. China PR: Ethical principles of human assisted reproductive technology and sperm banks. (Health Mo ed.; 2003.

12. Rosemann A, Luo H: Attitudes Towards the Donation of Human Embryos for Stem Cell Research Among Chinese IVF Patients and Students. J Bioeth Inq 2018, 15:441-457.

13. Wang Z, Wang S, Zhang $Y$, Jiang $X$ : Social media usage and online professionalism among registered nurses: A cross-sectional survey. Int J Nurs Stud 2019, 98:19-26.

14. Number of active WeChat messenger accounts Q2 2011-Q1 2021 [https://www.statista.com/statistics/255778/number-of-active-wechat-messenger-accounts/]

15. Nakhuda GS, Wang JG, Sauer MV: Posthumous assisted reproduction: a survey of attitudes of couples seeking fertility treatment and the degree of agreement between intimate partners. Fertil Steril 2011, 96:1463-1466.e1461.

16. Barton SE, Correia KF, Shalev S, Missmer SA, Lehmann LS, Shah DK, Ginsburg ES: Population-based study of attitudes toward posthumous reproduction. Fertil Steril 2012, 98:735-740.e735.

17. D Hans J: American Attitudes in Context: Posthumous Use of Cryopreserved Gametes. Journal of Clinical Research \& Bioethics 2013, 01.

18. Number of undergraduate students enrolled at public colleges and universities in China from 2009 to 2019. [https://www.statista.com/statistics/227028/number-of-students-at-universities-in-china/]

19. Start building a new powerful country in education [http://www.moe.gov.cn/jyb_xwfb/moe_176/202009/t20200910_486815.html]

20. Zeping Ren CX, Zhe Zhou: China Fertility Report 2019. Development Research 2019, 000:20-40.

21. Zhang M, Ying L-Y, Wu LH, Loke AY: The Experience of Chinese Couples Undergoing In Vitro Fertilization Treatment: Perception of the Treatment Process and Partner Support. Plos One 2015, 10.

22. Collins ME: The Impact of Infertility on Daily Occupations and Roles. J Reprod Infertil 2019, 20:24-34.

23. Vyas NM, Murugappan G, Westphal LM: Attitudes towards posthumous reproduction in patients undergoing embryo or gamete cryopreservation. Fertility and Sterility 2019, 111.

24. Simana S: Creating life after death: should posthumous reproduction be legally permissible without the deceased's prior consent? Journal of Law and the Biosciences 2018, 5:329-354.

25. Maddox N: Children of the Dead: Posthumous Conception, Critical Interests and Consent. J Law Med 2020, 27:645-662. 
26. Landau R: Posthumous sperm retrieval for the purpose of later insemination or IVF in Israel: an ethical and psychosocial critique. Hum Reprod 2004, 19:1952-1956.

27. Aziza-Shuster E: A child at all costs: posthumous reproduction and the meaning of parenthood. Hum Reprod 1994, 9:2182-2185.

28. G.Bahadur: Death and conception. Human Reproduction 2002, 17:2769-2775.

29. Krueger PM, Jutte DP, Franzini L, Elo I, Hayward MD: Family structure and multiple domains of child wellbeing in the United States: a cross-sectional study. Popul Health Metr 2015, 13:6.

30. Agnafors S, Bladh M, Svedin CG, Sydsjö G: Mental health in young mothers, single mothers and their children. BMC Psychiatry 2019, 19:112.

31. Weissenberg R, Landau R: Are two a family? Older single mothers assisted by sperm donation and their children revisited. Am J Orthopsychiatry 2012, 82:523-528.

32. Duriancik DM, Goff CR: Children of single-parent households are at a higher risk of obesity: A systematic review. J Child Health Care 2019, 23:358-369.

33. Kindregan CP Jr MM: Posthumous reproduction. Family Law Quarterly 2005, 35:579-597.

34. Lawson AK, Zweifel JE, Klock SC: Blurring the line between life and death: a review of the psychological and ethical concerns related to posthumous-assisted reproduction. Eur J Contracept Reprod Health Care 2016, 21:339-346.

\section{Figures}




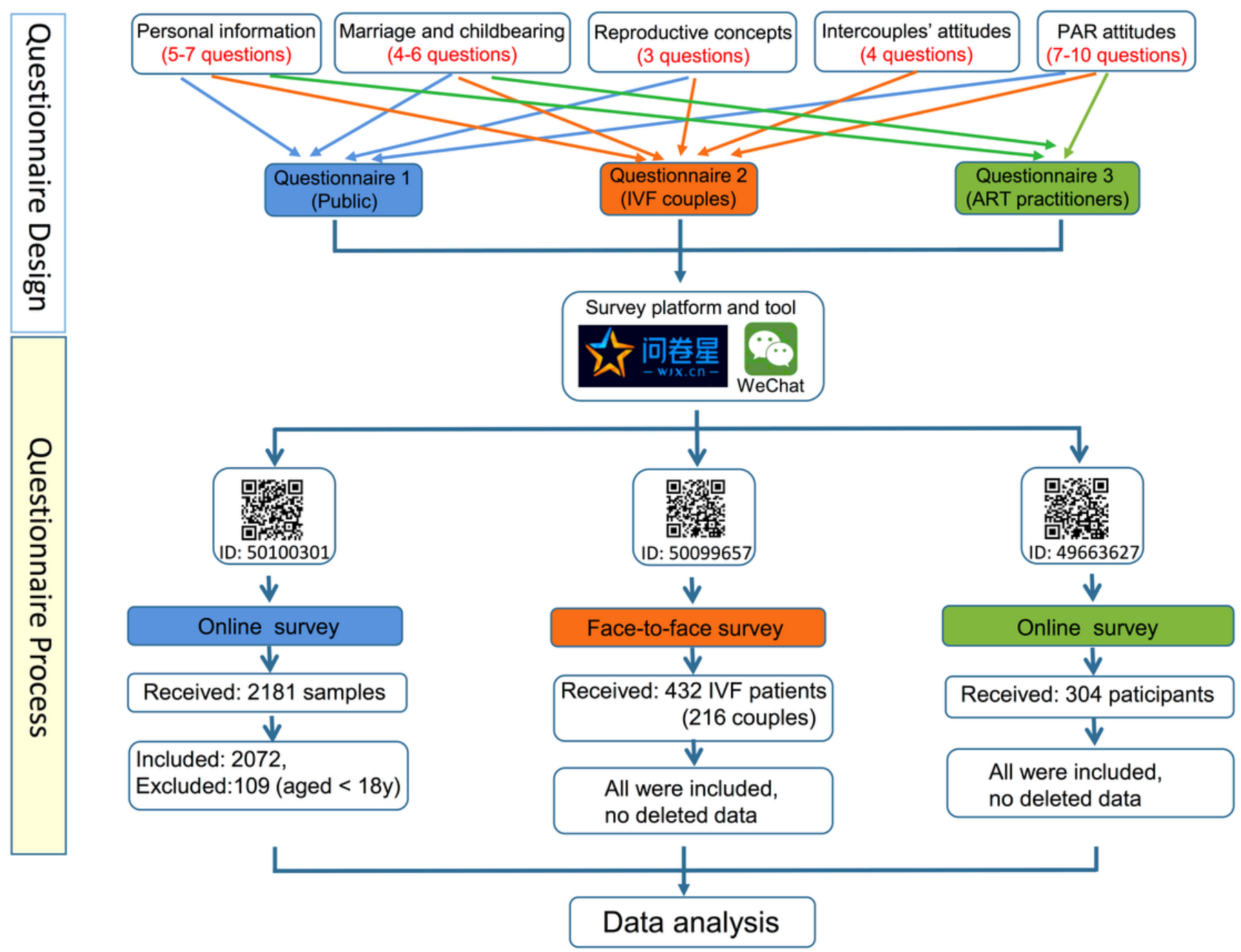

Figure 1

Flow chart of designing and processing the questionnaires. 
Public

$(\mathrm{N}=2076)$

A child is essential for a family

Yes

No

No opinion

Only boys inherit family line

Yes

No

No opinion
प

I
$52.46 \%(\mathrm{~N}=1089)$

$45.38 \%(\mathrm{~N}=942)$

$2.17 \%(\mathrm{~N}=45)$

$9.97 \%(\mathrm{~N}=207)$

$84.44 \%(\mathrm{~N}=1753)$

$5.59 \%(\mathrm{~N}=116)$
IVF couples

$(\mathrm{N}=432)$

Adopted children also inherit family line

Yes

No

No opinion

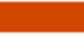

I

I

$89.58 \%(\mathrm{~N}=387)$

$8.56 \%(\mathrm{~N}=37)$

$1.85 \%(\mathrm{~N}=8)$

$34.26 \%(\mathrm{~N}=148)$

$51.39 \%(\mathrm{~N}=222)$

$14.35 \%(\mathrm{~N}=62)$
$53.81 \%(\mathrm{~N}=1117)$

$27.55 \%(\mathrm{~N}=572)$

$18.64 \%(\mathrm{~N}=387)$
$43.75 \%(\mathrm{~N}=189)$

$25.00 \%(\mathrm{~N}=108)$

$31.25 \%(\mathrm{~N}=135)$

Figure 2

Traditional reproductive viewpoints between Public and IVF patients. The length of the color bar represented the percentage.

\section{Public \\ $(\mathrm{N}=\mathbf{2 0 7 6})$}

PAR with a consent

Agree

disagree

No opinion

PAR without a consent

Agree

disagree

No opinion

Necessity of grieving

Yes

No

No opinion

Time for grieving (Year)

0.5-1

1-2

$\geq 2$

No

Mental health of offspring

Offspring health

Familiy bloodline

No opinion

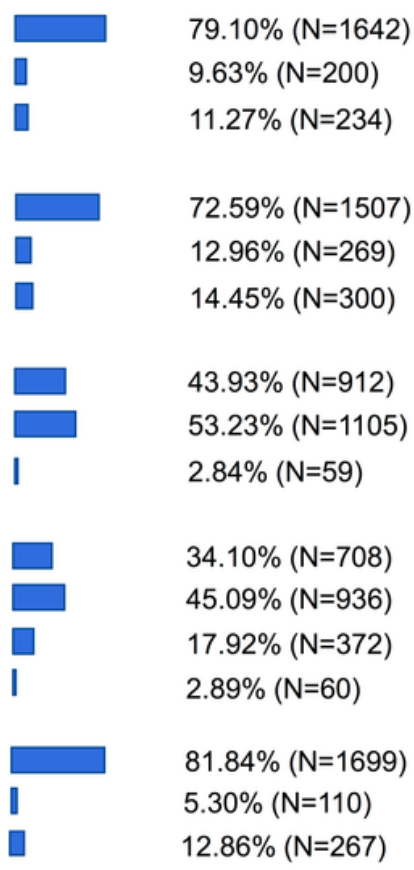

IVF couples

( $\mathrm{N}=432$ )

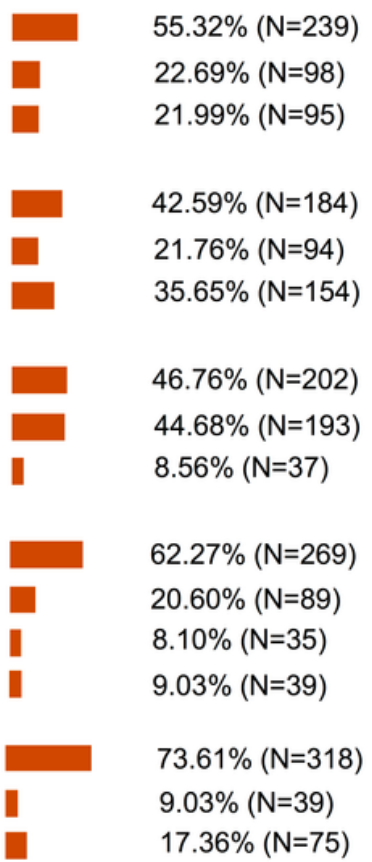

ART practitioners ( $N=304)$

$58.89 \%(\mathrm{~N}=179)$

$26.31 \%(\mathrm{~N}=80)$

$14.80 \%(\mathrm{~N}=45)$

$28.95 \%(\mathrm{~N}=88)$

$55.92 \%(\mathrm{~N}=170)$

$15.13 \%(\mathrm{~N}=46)$

$59.26 \%(\mathrm{~N}=168)$

$35.53 \%(\mathrm{~N}=108)$

$9.21 \%(\mathrm{~N}=28)$

$28.29 \%(\mathrm{~N}=86)$

$40.13 \%(\mathrm{~N}=122)$

$23.36 \%(\mathrm{~N}=71)$

$8.22 \%(\mathrm{~N}=25)$

$76.98 \%(\mathrm{~N}=234)$

$11.84 \%(\mathrm{~N}=36)$

$11.18 \%(\mathrm{~N}=34)$ 
Figure 3

Attitudes toward PAR-related issues in public, IVF patients, and ART practitioners. The length of the color bar represents the percentage. PAR: posthumous assisted reproduction. The complete sentence of the last question is "Inheriting family blood or ensuring offspring healthy grow-up, which do you think is more important?".

\section{Supplementary Files}

This is a list of supplementary files associated with this preprint. Click to download.

- STROBEchecklist.docx

- S1Instrumentforparticipants.docx

- S2PublicQuestionnaire.docx

- S3IVFcouplesquestionnaire.docx

- S4ARTpractitionersquestionnaire.docx

- SupplementalFigure1.tiff

- SupplementalFigure2.tiff 